ARTICLE

https://doi.org/10.1038/s41467-019-10949-9

\title{
Co-emergence of magnetic order and structural fluctuations in magnetite
}

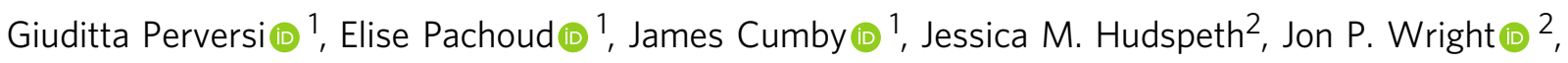
Simon A.J. $\mathrm{Kimber}^{3}$ \& J. Paul Attfield (1) ${ }^{1}$

The nature of the Verwey transition occurring at $T_{V} \approx 125 \mathrm{~K}$ in magnetite $\left(\mathrm{Fe}_{3} \mathrm{O}_{4}\right)$ has been an outstanding problem over many decades. A complex low temperature electronic order was recently discovered and associated structural fluctuations persisting above $T_{V}$ are widely reported, but the origin of the underlying correlations and hence of the Verwey transition remains unclear. Here we show that local structural fluctuations in magnetite emerge below the Curie transition at $T_{\mathrm{C}} \approx 850 \mathrm{~K}$, through $\mathrm{X}$-ray pair distribution function analysis. Around $80 \%$ of the low temperature correlations emerge in proportion to magnetization below $T_{\mathrm{C}}$. This confirms that fluctuations in Fe-Fe bonding arising from magnetic order are the primary electronic instability and hence the origin of the Verwey transition. Such hidden instabilities may be important to other spin-polarised conductors and orbitally degenerate materials.

\footnotetext{
${ }^{1}$ Centre for Science at Extreme Conditions (CSEC) and School of Chemistry, University of Edinburgh, Mayfield Road, Edinburgh EH9 3JZ, UK. ${ }^{2}$ European Synchrotron Radiation Facility, BP 156, 38042 Grenoble, France. ${ }^{3}$ ICB-Laboratoire Interdisciplinaire Carnot de Bourgogne, Université Bourgogne-Franche Comté, Bâtiment Sciences Mirande, 9 Avenue Alain, 21078 Dijon, France. Correspondence and requests for materials should be addressed to J.P.A. (email: j.p.attfield@ed.ac.uk)
} 
S hort-range structural correlations above the Verwey transition ${ }^{1-4}$ were reported in early diffuse scattering studies of magnetite ${ }^{5}$, and their persistence up to at least room temperature has been studied recently ${ }^{6}$ and corroborated by observations of anomalous phonon broadening 7 , a charge gap from optical and photoelectron spectroscopies 8,9 , and magnetic excitations driven by polaronic distortions in resonant inelastic $\mathrm{X}$-ray scattering data ${ }^{10}$. Analysis of the interatomic pair distribution function $(\mathrm{PDF})^{11}$ derived from total X-ray scattering data is a simple method for exploring local structure that is highly sensitive to the displacements of metal atoms associated with the formation of orbital molecules, metal-metal-bonded clusters such as the trimerons observed in magnetite ${ }^{12}$. Persistence of orbital molecules far above their long-range electronic ordering transitions has been discovered from X-ray PDF studies of $\mathrm{Li}_{2} \mathrm{RhO}_{4}{ }^{13}$, $\mathrm{Li}_{2} \mathrm{RuO}_{3}{ }^{14}, \mathrm{AlV}_{2} \mathrm{O}_{4}{ }^{15}$, and $\mathrm{GaV}_{2} \mathrm{O}_{4}{ }^{16}$.

The thermal variation of local structure in magnetite has been explored here over a wide temperature range encompassing both the Verwey and Curie transitions through synchrotron X-ray PDF analysis.

\section{Results}

Variable temperature PDF analysis of magnetite. Experimental details are in the "Methods" section. Total X-ray scattering data from a highly stoichiometric sample of magnetite were recorded from 90 to $923 \mathrm{~K}$. Representative scattering intensity $S(Q)$ plots and the derived PDFs $G(r)$ are shown in Fig. 1. The monoclinic superstructure adopted by magnetite below $T_{\mathrm{V}}$ is very complex with 168 small $(<0.24 \AA)$ displacements of atoms from their positions in the high temperature cubic spinel structure ${ }^{3}$. To fit the PDFs over all temperatures, we have used this monoclinic supercell with lattice parameters adjusted to a cubic metric and with each of the 168 atomic coordinates given by $p=p_{\mathrm{u}}+$ $f_{\mathrm{V}}\left(p_{\mathrm{d}}-p_{\mathrm{u}}\right)$ where $p_{\mathrm{u}}$ is the coordinate from an undistorted hightemperature cubic crystal structure refinement and $p_{\mathrm{d}}$ is the coordinate in the distorted $90 \mathrm{~K}$ structure reported previously ${ }^{3} \cdot f_{\mathrm{V}}$ is a Verwey shift parameter that describes the set of constrained structural displacements, such that $f_{\mathrm{V}}=0$ corresponds to the cubic spinel structure without any local distortions and $f_{\mathrm{V}}=1$ describes the full magnitude of distortions in the $90 \mathrm{~K}$ magnetite superstructure $^{3}$. Comparative views of the $f_{\mathrm{V}}=0$ and $f_{\mathrm{V}}=$ 1 structures are shown in ref. ${ }^{4}$. Three values of $f_{\mathrm{V}}$ were determined at each temperature by fitting the structural model to three successive regions of the PDF corresponding approximately to distances from atoms to their neighbours in the same unit cell (First Unit Cell range, covering all interatomic distances for $r<$ $9.36 \AA$ ) and similarly to atoms in the Second and Third Unit Cell regions. The structural models and fitting procedure are further described in "Methods", and fits to data and their sensitivity to changing $f_{\mathrm{V}}$ are shown in Fig. 2.

Thermal variations of refined structural quantities from the PDF fits are shown in Fig. 3. The cubic cell parameter and isotropic atomic temperature factors $U_{\text {iso }} s$ in Fig. 3a show a slight anomaly at the Verwey transition (previously found to be $T_{\mathrm{V}}=$ $123.4 \mathrm{~K}$ for this highly pure magnetite sample) ${ }^{17}$ but increase monotonically from 150 up to $923 \mathrm{~K}$. The Verwey shift $f_{\mathrm{V}}$ measures an averaged local structural distortion due to electronic fluctuations. The thermal variation of $f_{\mathrm{V}}$ when fitted to the Third Unit Cell separations (between atoms 16.8 to $24.6 \AA$ apart) in Fig. $3 \mathrm{~b}$ is a typical order-parameter behaviour at a first-order phase transition, with a sharp fall to $f_{\mathrm{V}}=0$ on warming through $T_{\mathrm{V}}$. Second Unit Cell correlations have a qualitatively similar variation although critical fluctuations decay more gradually above $T_{\mathrm{V}}$ and are estimated to persist up to $250-300 \mathrm{~K}$. However, structural correlations between atoms in the First Unit Cell range
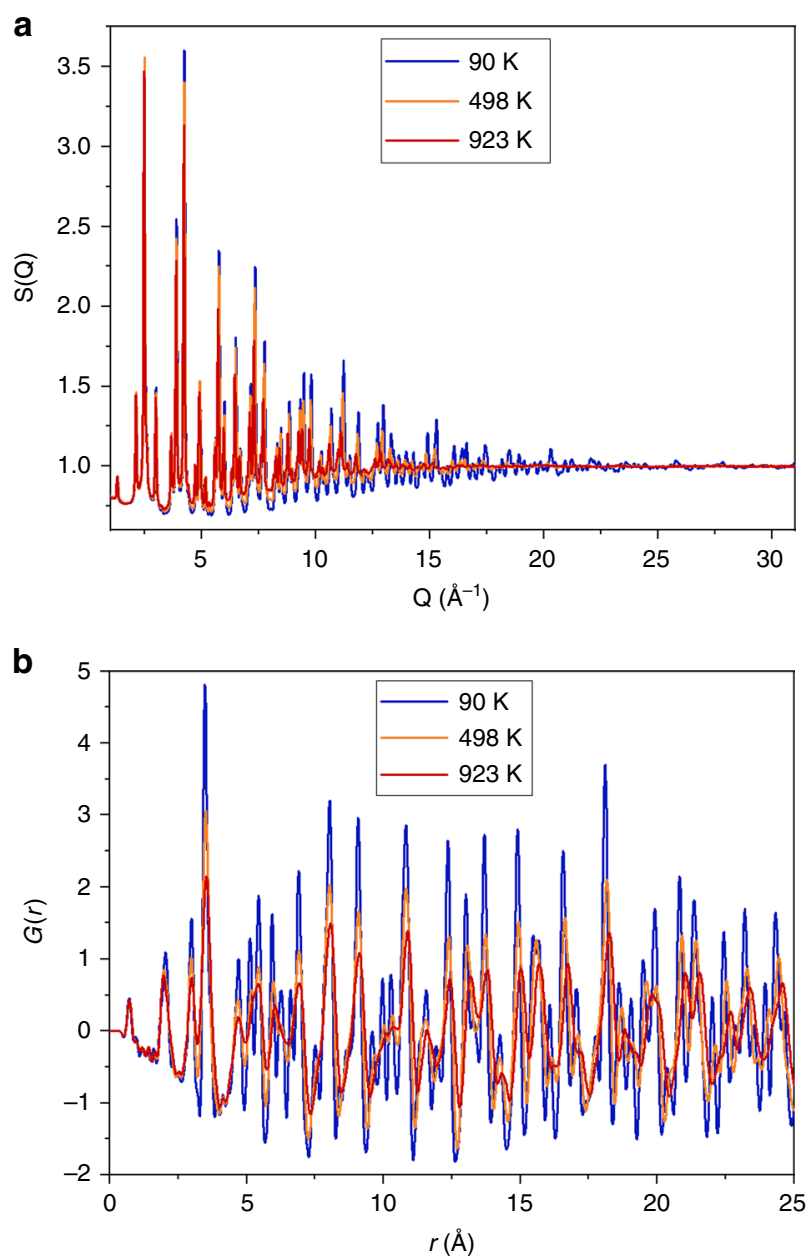

Fig. $1 X$-ray scattering data for magnetite. a Scattering intensities $S(Q)$ for magnetite at three representative temperatures. b Pair distribution functions $G(r)$ derived from $S(Q)$ data in a. Typical thermal effects leading to loss of high-Q features in $S(Q)$ and broadening of $G(r)$ peaks are observed

$(<9.4 \AA$ apart $)$ show a strikingly different behaviour. $f_{\mathrm{V}}$ falls a little on warming though the Verwey transition, but around $80 \%$ of the structural fluctuations remain in the cubic phase of magnetite up to $500 \mathrm{~K}$. At higher temperatures, $f_{\mathrm{V}}$ decreases rapidly to zero close to the Curie transition at $T_{\mathrm{C}} \approx 850 \mathrm{~K}$ and so behaves like an order parameter for the magnetic ordering transition with a very similar temperature dependence to the bulk magnetisation reported for a similar synthetic microcrystalline magnetite ${ }^{18}$. This is the key discovery of the present study as it demonstrates that the structural fluctuations responsible for the Verwey transition emerge directly with the long-range magnetic order below the Curie transition and scale with the magnetisation.

The thermal variations of the unit cell parameter and isotropic atomic temperature factors of magnetite plotted in Fig. 3a do not show any discontinuity at $T_{\mathrm{C}} \approx 850 \mathrm{~K}$, in keeping with previous high-temperature structural studies ${ }^{19,20}$. This demonstrates that our PDF fitting method is robust in decorrelating the overall lattice expansion and phonon motion, quantified by the latter parameters, from the local structural distortions due to electronic fluctuations described by $f_{\mathrm{V}}$. The magnitudes of the displacements of atoms from their ideal positions due to phonon motion and due to First Unit Cell electronic distortions are compared in Fig. 3c. The electronic distortion displacements are smaller than those due to thermal motion in the disordered high-temperature 

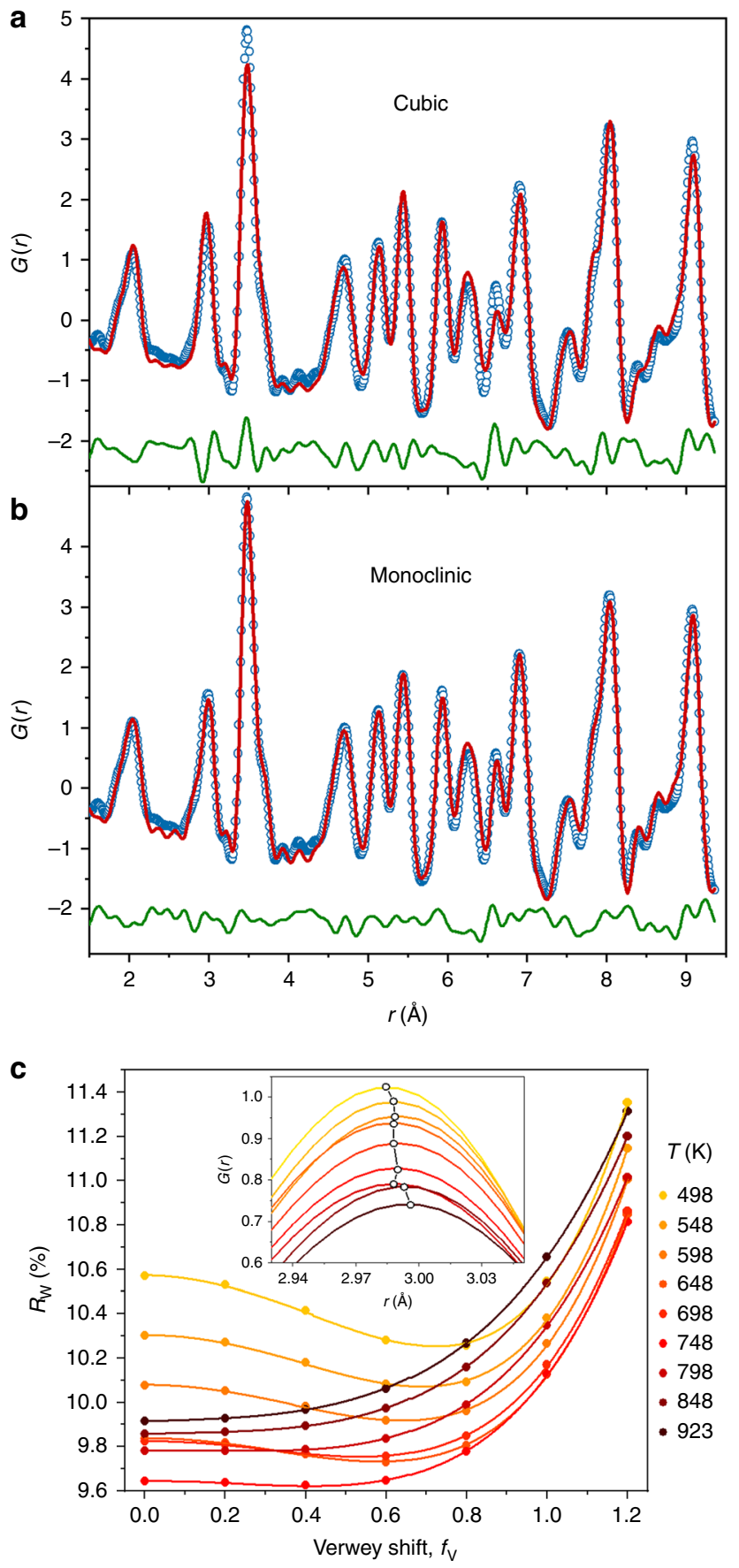

Fig. 2 Fitting of magnetite pair distribution functions (PDFs).

$\mathbf{a}, \mathbf{b}$ Comparison of fits of $\mathbf{a}$ the cubic (residual $R w=15.6 \%$ ) and $\mathbf{b}$ the monoclinic superstructure $(R \mathrm{w}=11.6 \%)$ models to the $90 \mathrm{~K}$ PDF. The visible improvement of the fit to the $r \approx 3 \AA$ peak measuring nearest neighbour $\mathrm{Fe}-\mathrm{Fe}$ (and $\mathrm{O}-\mathrm{O}$ ) distances and lowering of $\mathrm{Rw}$ demonstrates sensitivity to the monoclinic Verwey superstructure distortions. PDF data are open blue circles, fits are red curves and the difference is below in green. c $R \mathrm{w}$ as a function of the Verwey shift parameter $f_{\mathrm{V}}$ from fits to the First Unit Cell region of the high-temperature PDFs. The well-defined minimum seen at $f_{V} \approx 0.8$ for the $498 \mathrm{~K}$ fits becomes more shallow and moves to zero at the highest temperatures, as plotted in Fig. $3 \mathrm{~b}$. This subtle change of local structure can be seen in the shift in the maximum of the $3 \AA$ peak to slightly longer $r$ (inset), consistent with the loss of Fe-Fe distances shortened through trimeron bonding
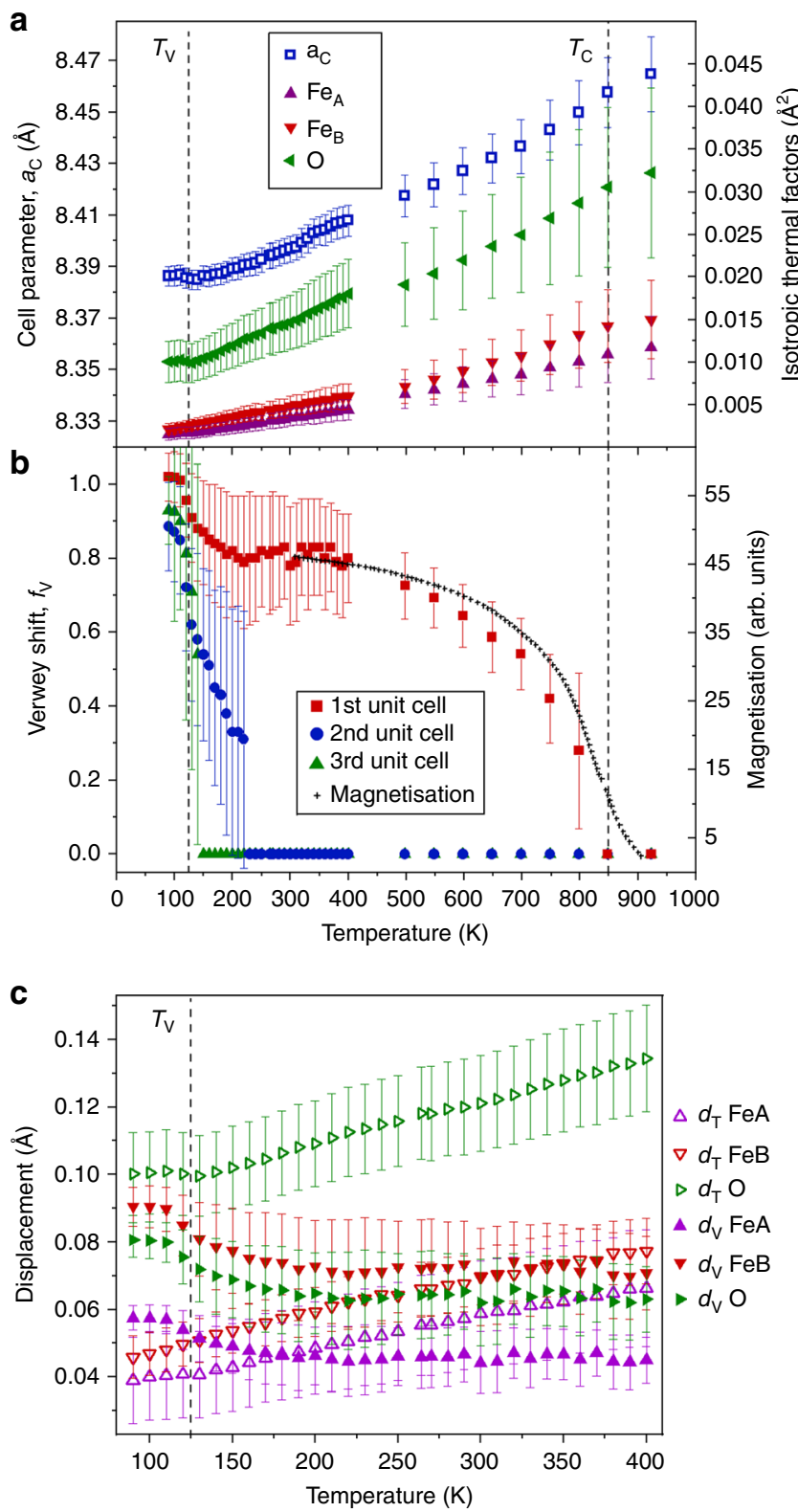

Fig. 3 Thermal variations of parameters from magnetite pair distribution function (PDF) fits. a Isotropic temperature factors $\mathrm{U}_{\text {iso }}$ for tetrahedral $\mathrm{Fe}_{\mathrm{A}}$, octahedral $\mathrm{Fe}_{\mathrm{B}}$, and oxygen sites and the cubic cell parameter from fits to the First Unit Cell PDF range. $\mathbf{b}$ Verwey shifts $f_{V}$ fitted to First, Second and Third Unit Cell PDF ranges. $f_{V}$ represents the magnitude of structural distortions due to electronic fluctuations in the cubic phase of magnetite above $T_{\mathrm{V}}$. The First Unit Cell values show that substantial local structural distortions persist up to $T_{C}$ and closely match the variation of bulk magnetisation data taken from ref. ${ }^{18}$. This demonstrates that the structural and electronic fluctuations responsible for the Verwey transition are a direct result of the long-range magnetic order. c Comparison of the atomic displacements due to electronic fluctuations $d_{\mathrm{V}}$, calculated from atomic coordinates for the First Unit Cell $f_{v}$ values, and those due to thermal motion taken as $d_{T}=\sqrt{ } U_{\text {iso }}$ from values shown in a. Error bars are estimated standard deviations from the refinements

region, but they increase to a comparable magnitude below $T_{\mathrm{V}}$. Although the timescale for these structural fluctuations is not directly measured, their lack of correlation with phonon motions suggests that they are essentially static or slowly diffusing. A recent quasi-elastic neutron scattering study of $\mathrm{GaV}_{2} \mathrm{O}_{4}$ spinel 
a

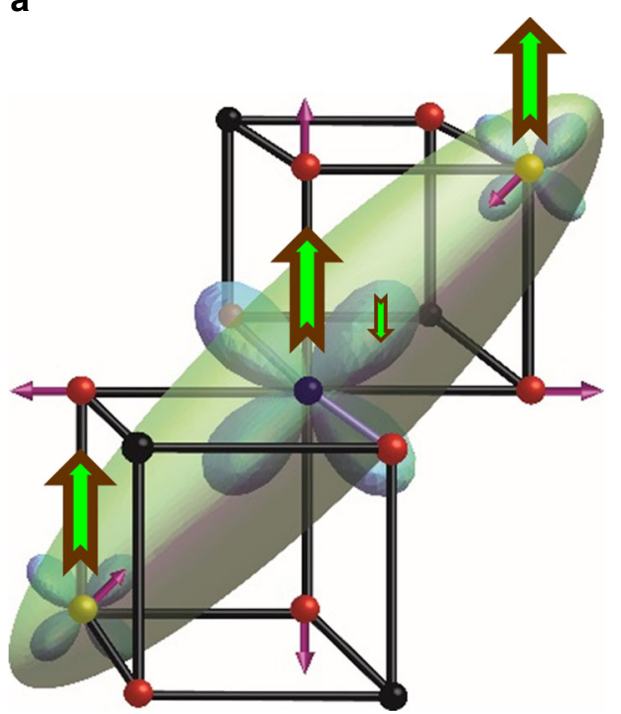

b

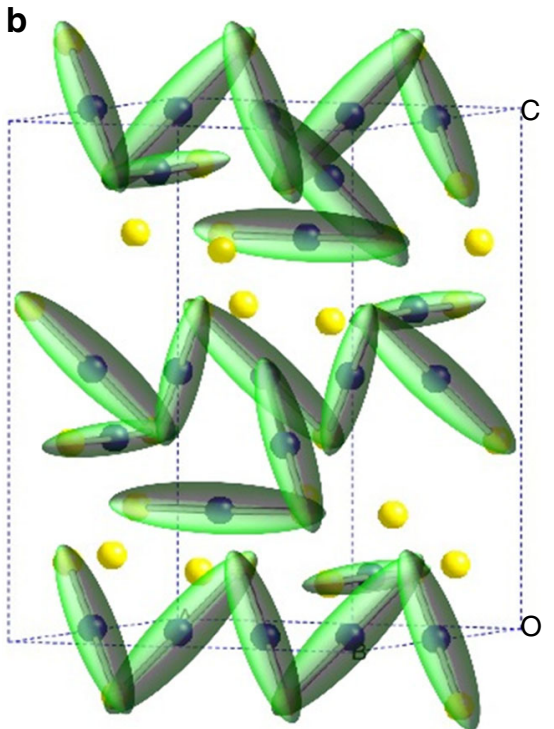

Fig. 4 Trimeron bonding driven by magnetic order in magnetite. Charge-ordered $\mathrm{Fe}^{2+} / \mathrm{Fe}^{3+}$ states are shown as blue/yellow spheres, trimerons are green, and oxide ions are red. a A single trimeron unit consisting of three Fe sites with parallel $S=5 / 2$ spins as shown by the brown-green arrows. Orbital order at the central $\mathrm{Fe}^{2+}$ site localises an antiparallel spin electron in one of the $t_{2 \mathrm{~g}}$ orbitals, which distorts the local structure through elongation of four Fe-O bonds and shortening of the distances through weak bonding to two Fe neighbours in the same plane, as indicated by the purple arrows. The minority spin electron density is approximated by the ellipsoid shown. b Long-range order of trimerons in the monoclinic superstructure formed below the Verwey transition. Corner-sharing of trimerons results in a complex pattern of atomic displacements that has been used to model the local structure in the pair distribution functions

above the 415-K charge-ordering transition found that structurally disordered orbital molecules remain well defined and without measurable dynamics up to $1100 \mathrm{~K}^{16}$.

Trimeron fluctuations in cubic magnetite. The complex monoclinic superstructure of magnetite below $T_{\mathrm{V}}$ was previously found to exhibit trimerons (Fig. 4a), small polarons surrounding three $\mathrm{Fe}$ units that share corners with each other to build up a long-range ordered network as shown in Fig. 4b. The end-to-end $\mathrm{Fe}-\mathrm{Fe}$ distance in a trimeron is $6 \AA$, so their local lattice distortion lies within the First Unit Cell range $(<9.4 \AA)$ and hence arises below the Curie transition as demonstrated in Fig. 3b. Persistence of a disordered glassy network of trimerons in the cubic phase of magnetite up to $T_{\mathrm{C}}$ provides a mechanism for the coupling of the high-temperature structural fluctuations to the magnetism and hence for the origin of the Verwey transition as follows.

Trimerons result from coupled $\mathrm{Fe}^{2+} / \mathrm{Fe}^{3+}$ charge ordering, $\mathrm{Fe}^{2+}$ orbital ordering, and weak $\mathrm{Fe}-\mathrm{Fe}$ bonding effects driven by ferromagnetism within three $\mathrm{Fe}$ atom units as described in the caption of Fig. $4 \mathrm{a}^{3}$. Comparison with other mixed valent $\mathrm{Fe}^{2+} / \mathrm{Fe}^{3+}$ oxides shows that neither the charge nor orbital orders require long-range spin order, as evidenced by $\mathrm{Fe}_{2} \mathrm{OBO}_{3}{ }^{21,22}$ and $\mathrm{LuFe}_{2} \mathrm{O}_{4}{ }^{23}$, both of which have charge and orbital ordering transitions at higher temperatures than their magnetic transitions. However, the weak bonding interactions that shorten $\mathrm{Fe}-\mathrm{Fe}$ distances in the trimerons do require ferromagnetic alignment of the three core $S=5 / 2$ spins, as demonstrated recently in $\mathrm{CaFe}_{3} \mathrm{O}_{5}$ where phase-separated trimeron and non-trimeron ground states are observed ${ }^{24}$. Hence, the rapid emergence of structural fluctuations in proportion to magnetisation on cooling below the long-range magnetic ordering temperature confirms that the direct $\mathrm{Fe}-\mathrm{Fe}$ bonding interactions are the primary driver of the local distortions in magnetite. As suggested previously, the $\mathrm{Fe}-\mathrm{Fe}$ interactions induce associated charge and orbital fluctuations that become ordered as trimeron units below the Verwey transition ${ }^{3}$. The regular cubic spinel arrangement in which all nearestneighbour $\mathrm{Fe}-\mathrm{Fe}$ distances are equivalent is thus unstable with respect to local variations in $\mathrm{Fe}-\mathrm{Fe}$ separations in the magnetically ordered state of magnetite. There are many degenerate arrangements for linking trimerons with similar connectivities to the observed low-temperature network shown in Fig. $4 b^{3}$, so the long-range electronic order is frustrated. The ratio of energy scales for formation $\left(T_{\mathrm{C}}\right)$ and long-range order $\left(T_{\mathrm{V}}\right)$ of the electronic distortions serves as a measure of the degree of electronic frustration, analogous to the Weiss to Néel temperature ratio used for frustrated antiferromagnets. The value of $T_{\mathrm{C}} / T_{\mathrm{V}} \approx 7$ confirms that electronic order in magnetite is strongly frustrated.

\section{Discussion}

Analysis of the PDF derived from X-ray scattering data reveals that local structural distortions due to electronic fluctuations emerge at the Curie transition of magnetite. This confirms that fluctuations in $\mathrm{Fe}-\mathrm{Fe}$ bonding are the dominant electronic instability that couples charge and orbital fluctuations to the magnetic order and hence are the origin of the Verwey transition. Although many experimental studies of magnetite have been carried out at temperatures around $T_{\mathrm{V}}$, changes near $T_{\mathrm{C}}$ are much less investigated and will be important to explore the incipient electronic fluctuations further. Such 'hidden' local instabilities may also be important to the physics of other spin-polarised conductors and orbitally degenerate materials, and the assumed uniformity of their high temperature structures may require critical reassessment.

\section{Methods}

Powder $\mathbf{X}$-ray scattering experiments. The same highly pure magnetite powder $\left(\mathrm{Fe}_{3-\delta} \mathrm{O}_{4}\right.$ with $\left.\delta<0.0001\right)$ as used in previous studies ${ }^{3,25-27}$ was packed in a $0.5-\mathrm{mm}$ diameter quartz capillary and sealed under Ar atmosphere in a glove bag. Preliminary measurements were performed on beam line ID15B at ESRF, Grenoble and full data were collected on ESRF instrument ID11 with wavelength $\lambda=0.15720$ (1) Å using a FReLoN camera for diffraction pattern acquisition. A nitrogen cryostream was used to collect patterns between 90 and $400 \mathrm{~K}$ in $10 \mathrm{~K}$ steps. Small anomalies seen in several refined quantities around 250-300 K in Fig. 3 most likely reflect ice in the sample vicinity leading to additional scattering contributions, as magnetite does not have an intrinsic lattice anomaly in this temperature region. A 
hot air blower was used to collect data between 498 and $923 \mathrm{~K}$ in $50 \mathrm{~K}$ steps. Empty capillary data were collected at $90,200,473,573,673,773$, and $873 \mathrm{~K}$. For each data set, the temperature was stabilised for $10 \mathrm{~min}$ and then data were collected for 10 min using the accumulation mode of the detector for 10-s exposure frames. Data were also collected from $\mathrm{CeO}_{2}$ and $\mathrm{Si}$ standards at $300 \mathrm{~K}$ to calibrate the instruments and determine instrumental parameters.

Instrument calibration and image integration was performed with the pyFAI software ${ }^{28}$. The instrument model was recalibrated for every temperature in the cryostat data sets in order to allow for slight detector shifts. A fixed instrument model was used for the hot air blower data. Data sets were converted to scattering intensities $S(Q)$ as a function of scattering vector $Q$ and these were transformed to PDFs $G(r)$, where $r$ is interatomic distance, using the pdfgetx 3 suite $^{29}$. Parameters values $Q_{\min }=1 \AA^{-1}, Q_{\max }=31 \AA^{-1}$, and $r_{\text {poly }}=0.9 \AA$ were applied to all data sets to enable structural changes in $G(r)$ to be analysed consistently across the full temperature range. Representative $S(Q)$ and $G(r)$ plots are shown in Fig. 1 and Supplementary Fig. 1.

Structural models for PDF analysis. Structural models used to fit magnetite PDFs at all temperatures were based on the supercell of the low-temperature structure with monoclinic space group $C c$ symmetry, which contains 56 unique atoms with 168 variable coordinates. A cubic model previously refined against $130 \mathrm{~K}$ powder $\mathrm{X}$-ray and neutron data ${ }^{25,26}$ was used as the reference undistorted structure. The VESTA software ${ }^{30}$ was used to generate a supercell of the cubic structure with metric $a=b=\sqrt{ } 2 a_{\mathrm{c}}, c=2 a_{\mathrm{c}}, \alpha=\beta=\gamma=90^{\circ}$, where $a_{\mathrm{c}}$ is the cubic spinel cell parameter, and atomic coordinates $p_{\mathrm{u}}$ in $C c$ space group symmetry are shown in Supplementary Table 1 . This model has $f_{\mathrm{V}}=0$. The previously reported $90 \mathrm{~K}$ monoclinic $C c$ structure $^{3}$ was transformed to the same cell metric (from $a=$ 11.88881(3) $\AA, b=11.84940(3) \AA ̊, c=16.77515(1) \AA ⿻, \beta=90.2363(2)^{\circ}$ to $a=b=$ 11.86182(3) $\AA, c=16.77515(1) \AA, \beta=90^{\circ}$ ). Atomic positions were shifted slightly in order to preserve the magnitude of the distortions as generated by the ISODISTORT program ${ }^{31}$. This approximation was found to have little effect on fits of the $C c$ model to the $90 \mathrm{~K} \mathrm{PDF}(R \mathrm{w}=11.5 \%$ for monoclinic cell parameters vs. $R \mathrm{w}=11.6 \%$ with metric constraints). Coordinates $p_{\mathrm{d}}$ for this fully distorted reference structure $\left(f_{\mathrm{V}}=1\right)$ are shown in Supplementary Table 2 . To vary the magnitude of the structural distortion, seven structural models were constructed by taking linear combinations of the undistorted and distorted coordinates $p=p_{\mathrm{u}}+$ $f_{\mathrm{V}}\left(p_{\mathrm{d}}-p_{\mathrm{u}}\right)$ for values of the Verwey shift $f_{\mathrm{V}}$ from 0 to 1.2 in increments of 0.2 .

PDF fits. Refinements of structural models including simulation of termination ripples were performed with the PDFgui software ${ }^{32}$. A fit to the $\mathrm{CeO}_{2} \mathrm{PDF}$ with a fixed structural model was used to extract the instrument-sensitive parameters $Q_{\text {damp }}=0.0475(4) \AA^{-1}$ and $Q_{\text {broad }}=0.0186(3) \AA^{-1}$. It was not possible to minimise the value of $f_{\mathrm{V}}$ directly in the refinements and so the best-fit value was found by comparing $R \mathrm{ws}$ for the seven structural models with varying fixed $f_{\mathrm{V}}$ values.

A 'box-car' refinement procedure was followed where, at each temperature, each of the seven structural models were fitted to three regions of the PDF; $r=$ $1.50-9.36,9.30-16.85$, and $16.80-24.60 \AA$, to fit correlations between atoms separated by approximate First, Second, and Third Unit Cell distances, respectively. The cubic cell parameter $a_{\mathrm{c}}$, separate isotropic thermal parameters $U_{\text {iso }}$ for tetrahedrally coordinated $\mathrm{Fe}_{\mathrm{A}}$, octahedral $\mathrm{Fe}_{\mathrm{B}}$, and $\mathrm{O}$ sites, and the peak width correlation parameter $\delta_{1}$ were refined during fits to data in the First Unit Cell range. Second and Third Unit Cell fits used $a_{\mathrm{c}}$ values from the First Unit Cell fits, and $\delta_{1}$ was set to 0 . A further constraint of equal $\mathrm{Fe}_{\mathrm{A}}$ and $\mathrm{Fe}_{\mathrm{B}} U_{\mathrm{iso}}$ s was needed to fit Third Unit Cell data sets for temperatures $>150 \mathrm{~K}$. Fits to $90 \mathrm{~K}$ First Unit Cell data are shown in Fig. 3a.

The best-fit value of $f_{\mathrm{V}}$ for each temperature and data range was found from the position of the minimum in the $R \mathrm{w}$ vs. $f_{\mathrm{V}}$ curve fitted by the arbitrary function:

$$
R w=\left(A f_{\mathrm{V}}^{2}+B f_{\mathrm{V}}+C\right) \cdot \exp \left(D f_{\mathrm{V}}\right)
$$

where $A, B, C$, and $D$ are refined parameters. Fits to $R \mathrm{w}$ vs. $f_{\mathrm{v}}$ points are shown in Fig. $2 \mathrm{c}$ and Supplementary Fig. 2. Estimated standard deviations (esds) in the bestfit $f_{\mathrm{V}}$ values were calculated from those in $A, B, C$, and $D$ in Eq. (1). The esds on $f_{\mathrm{V}}$ values $<0.3$ are large so these $f_{\mathrm{V}}$ values were set to zero. The best-fit First Unit Cell's cell parameters and $U_{\text {isso }}$ and their errors were calculated by linear interpolation between values at the two $f_{\mathrm{V}}$ increments closest to the minimum value. Lattice parameters were also fitted to the diffraction data using the Rietveld method and are shown in Supplementary Fig. 3. An approximate correlation length for the structural distortions was calculated from the First Unit Cell $f_{\mathrm{V}}$ values and is shown in Supplementary Fig. 4.

\section{Data availability}

Data that support the findings of this study have been deposited at https://doi.org/ $10.7488 / \mathrm{ds} / 2574$.

Received: 15 April 2019 Accepted: 10 June 2019

Published online: 28 June 2019

\section{References}

1. Verwey, E. J. W. Electronic conduction of magnetite $\left(\mathrm{Fe}_{3} \mathrm{O}_{4}\right)$ and its transition point at low temperatures. Nature 144, 327-328 (1939).

2. Walz, F. The Verwey transition-a topical review. J. Phys.Condens. Matter 14, R285-R340 (2002).

3. Senn, M. S., Wright, J. P. \& Attfield, J. P. Charge order and three-site distortions in the Verwey structure of magnetite. Nature 481, 173-176 (2012).

4. Senn, M. S., Wright, J. P. \& Attfield, J. P. The Verwey phase of magnetite-a long-running mystery in magnetism. J. Korean Phys. Soc. 62, 1372-1375 (2013).

5. Shapiro, S. M., Iizumi, M. \& Shirane, G. Neutron scattering study of the diffuse critical scattering associated with the Verwey transition in magnetite $\left(\mathrm{Fe}_{3} \mathrm{O}_{4}\right)$. Phys. Rev. B 14, 200-207 (1976).

6. Bosak, A. et al. Short-range correlations in magnetite above the Verwey temperature. Phys. Rev. X 4, 011040 (2014).

7. Hoesch, M. et al. Anharmonicity due to electron-phonon coupling in magnetite. Phys. Rev. Lett. 110, 207204 (2013)

8. Park, S. K., Ishikawa, T. \& Tokura, Y. Charge-gap formation upon the Verwey transition in $\mathrm{Fe}_{3} \mathrm{O}_{4}$. Phys. Rev. B 58, 3717 (1998).

9. Taguchi, M. et al. Temperature dependence of magnetically active charge excitations in magnetite across the Verwey transition. Phys. Rev. Lett. 115, 256405 (2015).

10. Huang, H. Y. et al. Jahn-Teller distortion driven magnetic polarons in magnetite. Nat. Commun. 8, 15929 (2017).

11. Egami, T. \& Billinge, S. J. L. Underneath the Bragg Peaks: Structural Analysis of Complex Materials, Vol. 16 (Pergamon Materials Series, Pergamon Press, Oxford, 2012)

12. Attfield, J. P. Orbital molecules in electronic materials. APL Mater. 3, 041510 (2015).

13. Knox, K. R. et al. Local structural evidence for strong electronic correlations in spinel $\mathrm{LiRh}_{2} \mathrm{O}_{4}$. Phys. Rev. B 88, 174114 (2013).

14. Kimber, S. A. et al. Valence bond liquid phase in the honeycomb lattice material $\mathrm{Li}_{2} \mathrm{RuO}_{3}$. Phys. Rev. B 89, 081408 (2014).

15. Browne, A. J., Kimber, S. A. J. \& Attfield, J. P. Persistent three-and four-atom orbital molecules in the spinel $\mathrm{AlV}_{2} \mathrm{O}_{4}$. Phys. Rev. Mater. 1, 052003 (2017).

16. Browne, A. J., Lithgow, C., Kimber, S. A. J. \& Attfield, J. P. Orbital molecules in the new spinel $\mathrm{GaV}_{2} \mathrm{O}_{4}$. Inorg. Chem. 57, 2815-2822 (2018).

17. Senn, M. S., Wright, J. P., Cumby, J. \& Attfield, J. P. Charge localization in the Verwey structure of magnetite. Phys. Rev. B 92, 024104 (2015).

18. Ponomar, V. P., Dudchenko, N. O. \& Brik, A. B. Thermal stability of microand nanoscale magnetite by thermomagnetic analysis data. In 2017 IEEE 7th International Conference Nanomaterials: Application \& Properties (NAP), Odessa, 02MFPM03, Ed. Pogrebnjak, Alexander D., IEEE, Piscataway, NJ, USA (2017)

19. Okudera, H., Kihara, K. \& Matsumoto, T. Temperature dependence of structure parameters in natural magnetite: single crystal X-ray studies from 126 to 773 K. Acta Cryst. B 52, 450-457 (1996).

20. Levy, D., Giustetto, R. \& Hoser, A. Structure of magnetite $\left(\mathrm{Fe}_{3} \mathrm{O}_{4}\right)$ above the Curie temperature: a cation ordering study. Phys. Chem. Miner. 39, 169-176 (2012).

21. Attfield, J. P. et al. Electrostatically driven charge-ordering in $\mathrm{Fe}_{2} \mathrm{OBO}_{3}$. Nature 396, 655-658 (1998)

22. Angst, $M$. et al. Charge order superstructure with integer iron valence in $\mathrm{Fe}_{2} \mathrm{OBO}_{3}$. Phys. Rev. Lett. 99, 086403 (2007).

23. de Groot, J. et al. Charge order in $\mathrm{LuFe}_{2} \mathrm{O}_{4}$ : an unlikely route to ferroelectricity. Phys. Rev. Lett. 108, 187601 (2012).

24. Hong, K. H., Arevalo-Lopez, A. M., Cumby, J., Ritter, C. \& Attfield, J. P. Long range electronic phase separation in $\mathrm{CaFe}_{3} \mathrm{O}_{5}$. Nat. Commun. 9, 2975 (2018).

25. Wright, J. P., Attfield, J. P. \& Radaelli, P. G. Long range charge ordering in magnetite below the Verwey transition. Phys. Rev. Lett. 87, 266401 (2001).

26. Wright, J. P., Attfield, J. P. \& Radaelli, P. G. Charge ordered structure of magnetite $\mathrm{Fe}_{3} \mathrm{O}_{4}$ below the Verwey transition. Phys. Rev. B 66, 214422 (2002).

27. Goff, R. J., Wright, J. P., Attfield, J. P. \& Radaelli, P. G. Resonant X-ray diffraction study of the charge ordering in magnetite. J. Phys. Condens. Matter 17, 7633-7642 (2005).

28. Kieffer, J. \& Wright, J. P. PyFAI: a Python library for high performance azimuthal integration on GPU. Powder Diffr. 28, S339-S350 (2013).

29. Juhás, P., Davis, T., Farrow, C. L. \& Billinge, S. J. PDFgetX3: a rapid and highly automatable program for processing powder diffraction data into total scattering pair distribution functions. J. Appl. Crystallogr. 46, 560-566 (2013).

30. Momma, K. \& Izumi, F. VESTA: a three-dimensional visualization system for electronic and structural analysis. J. Appl. Crystallogr. 41, 653-658 (2008).

31. Campbell, B. J., Stokes, H. T., Tanner, D. E. \& Hatch, D. M. ISODISPLACE: an internet tool for exploring structural distortions. J. Appl. Cryst. 39, 607-614 (2006).

32. Farrow, C. L. et al. PDFfit 2 and PDFgui: computer programs for studying nanostructure in crystals. J. Phys. Condens. Matter 19, 335219 (2007). 


\section{Acknowledgements}

We acknowledge financial support from European Research Council (ERC) and Science and Technology Facilities Council (STFC) for provision of beam time at ESRF. This work was also supported by the French "Investissements d'Avenir" program, project ISITEBFC (contract ANR-15-IDEX-0003). We thank Professor J.M. Honig (Purdue

University) for provision of the high-quality magnetite sample.

\section{Author contributions}

G.P., E.P., J.C., S.A.J.K. and J.P.A. designed the concept for this study. G.P., E.P., J.C. J.M.H., J.P.W. and S.A.J.K., performed the experimental work and data analysis. G.P. and J.P.A. wrote the manuscript with inputs from all the authors.

\section{Additional information}

Supplementary Information accompanies this paper at https://doi.org/10.1038/s41467019-10949-9.

Competing interests: The authors declare no competing interests.

Reprints and permission information is available online at http://npg.nature.com/ reprintsandpermissions/
Peer review information: Nature Communications thanks Sverre Selbach for his contribution to the peer review of this work.

Publisher's note: Springer Nature remains neutral with regard to jurisdictional claims in published maps and institutional affiliations.

cc (i) Open Access This article is licensed under a Creative Commons Attribution 4.0 International License, which permits use, sharing, adaptation, distribution and reproduction in any medium or format, as long as you give appropriate credit to the original author(s) and the source, provide a link to the Creative Commons license, and indicate if changes were made. The images or other third party material in this article are included in the article's Creative Commons license, unless indicated otherwise in a credit line to the material. If material is not included in the article's Creative Commons license and your intended use is not permitted by statutory regulation or exceeds the permitted use, you will need to obtain permission directly from the copyright holder. To view a copy of this license, visit http://creativecommons.org/ licenses/by/4.0/.

(C) The Author(s) 2019 